\title{
Hypermedia in Radiology: Computer-Assisted Education
}

\author{
Charles C. Chen, Paul B. Hoffer, and Henry A. Swett
}

\begin{abstract}
Hypertext is a new computer-based method of presenting information that provides greater flexibility than conventional methods of continuing education. With a hypertext system, an individual using the computer can acquire more information on a word or concept that needs to be pursued in depth. Hypermedia is an expanded concept which uses the computer's ability to incorporate images, sounds, and video imeges in addition to text. This interective, multimedia approach customizes information for more effective learning. The authors devised a prototype hypermedia textbook of nuclear medicine using a personal computer with hypermedia software that contains text. graphs, tables, figures, literature citations, and an easily perusable image database. All the information is organized with multiple cross-references, allowing instant branching to relevant facts, in different levels of detail. The system's applications and the ease of expansion or modification by the user are described.
\end{abstract}

(1) 1989 by W.B. Saunders Company

KEYWORDS: Hypermedia, computer-assisted education, computer database.

$\mathbf{I}^{\mathrm{N}}$ THE PAST few years, there has been an exponential expansion in the volume of medical knowledge. Growth of new imaging modalities such as computed tomography, ultrasound, magnetic resonance imaging, and nuclear medicine make it increasingly difficult for radiologists to practice state of the art radiology. Residency programs have been forced to extend the number of years of training. Board exams must cover a wider range of topics.

Different media for displaying image information are required for various modalities. For example, Doppler ultrasound information is enhanced by the addition of sound; ultrasound and nuclear cardiology requires media that are able to display dynamic images in order to describe motion abnormalities. Multiple projection or tomographic images are required in order to represent three-dimensional informa-

From the Yale University School of Medicine, New Haven, $C T$.

Address reprint requests to Paul B. Hoffer, MD. Director of Nuclear Medicine, Yale University School of Medicine, 333 Cedar St, Room TE-2, New Haven, CT 06510.

( 1989 by W.B. Saunders Company.

0897-1889/89/0201-0002\$03.00/0 tion. In the words of Marshall McLuhan, "the medium is the message."

Many continuing education materials are available to deal with these problems, but each has limitations. Radiologists have different depths of knowledge of each subject, and information provided by conventional methods may be either too superficial or too detailed for an individual user. Computer-assisted techniques have the potential of tailoring the information to each individual.

Text and images can be supplied by textbooks and journals, and videotape can display dynamic image information. All of these methods present information in a linear format. They are cost effective, but lack the ability to adequately represent complex information.

During radiology training, a great deal of knowledge is transmitted via spoken words, slides, and films. Residents learn from interactive communication with more experienced radiologists. In order to interpret images, these experienced radiologists help students and residents to correlate a variety of information in a coherent manner. A radiologist must learn to associate findings on radiographs with clinical information (the patient's history, physical, and test results), knowledge of anatomy, pathophysiology, epidemiology, and alternate methods of diagnosis. eg, other imaging modalities or biopsy. While this complex thought process may be taught in textbooks that are written in a linear format, a nonlinear presentation of this information may be more natural and effective.

Hypertext is a computer-assisted method of communicating information that provides greater flexibility than conventional methods. Hypertext is also known as nonlinear text, "a combination of natural language text with the computer's capacity for interactive branching, or dynamic dispiay . . of a nonlinear text . . . which cannot be printed conveniently on a conventional page." An extension of hypertext is the more general concept of hypermedia, in which the computer incorporates static images, video images, and digitized sound into text.

The authors developed a prototype nuclear medicine hypermedia textbook or "hypertext- 


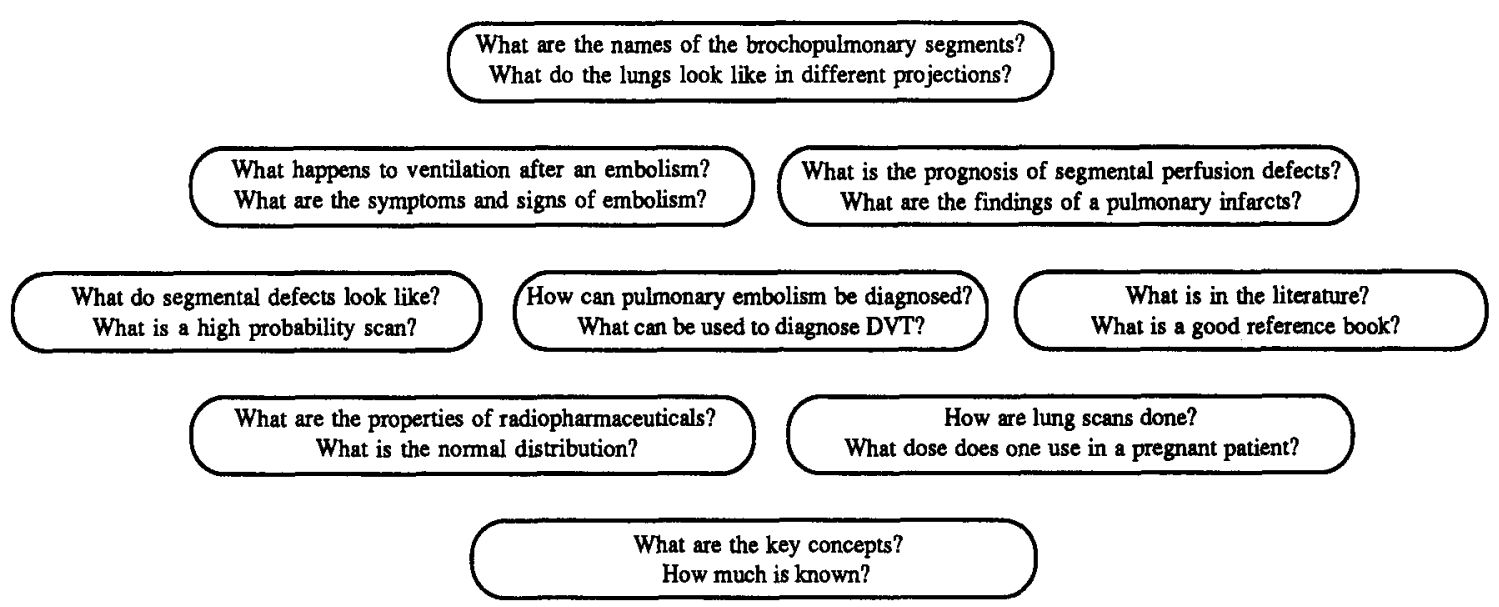

Fig 1. The radiologic thought associations needed to interpret a lung scan.

book" to investigate the potential of hypermedia computer technology in teaching diagnostic radiology. This overview will examine the advantages, disadvantages, and utility of digitized information organized in a nonlinear hypermedia format.

\section{HYPERMEDIA CHARACTERISTICS}

What distinguishes hypertext from other formats is its ability to perform high speed links on textual information. It is "a computer-based medium for thinking and communication." ${ }^{3}$ Figure 1 depicts some radiologic thought associations needed to interpret findings from an image. Hypertext closely simulates the radiologist's associative learning process, and can improve the quality and flexibility of computer-assisted instruction (compare Figs 1 and 2).

Although computer-supported links are the essence of hypermedia, the actual information is presented with text and graphics on a series of structured computer windows, similar to information put on $3 \times 5$ notecards. These cards may be shuffled and linked in unlimited ways.

\section{APPLICATION OF HYPERMEDIA}

Many hypertext systems have been described elsewhere. ${ }^{2}$ The authors used commercially available software (HyperCard, Apple Computer, Cupertino, CA) to create a nuclear medicine interactive textbook, or "hypertextbook."3 The hardware consists of a microcomputer (Apple Macintosh II, Apple Computer) with a hard disk drive and a computer mouse.

Text, graphs, tables, and illustrations were assembled from standard textbooks and journals. Images and illustrations were collected by using digitizers. Figure 2 illustrates the basic structure of this prototype nuclear medicine hypertextbook. Each piece of information is linked to other pieces of information, and the organization is nonlinear. The user can also easily modify the way pieces of information are linked by changing existing links or creating new links.

Figure 3 shows an overview of a portion of the nuclear medicine hypertextbook. Figures 4 to 7 demonstrate a simple search through the hypertextbook. Figure 4 shows the "Main Menu." By activating the "Anatomy" button on the "Main Menu" with the mouse, the "Anatomy" window

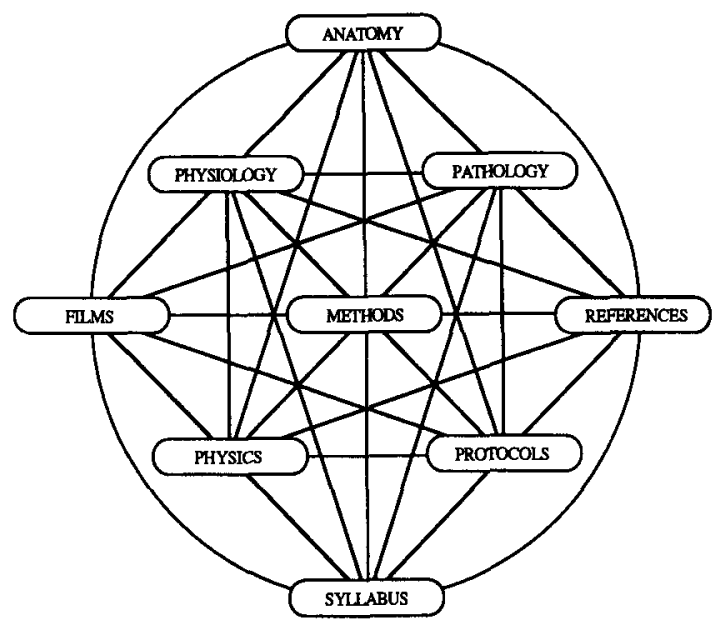

Fig 2. Structure of a prototype nuclear medicine hypertextbook. The lines represent the links between different types of information. The structure is both lineer and nonlinear. 


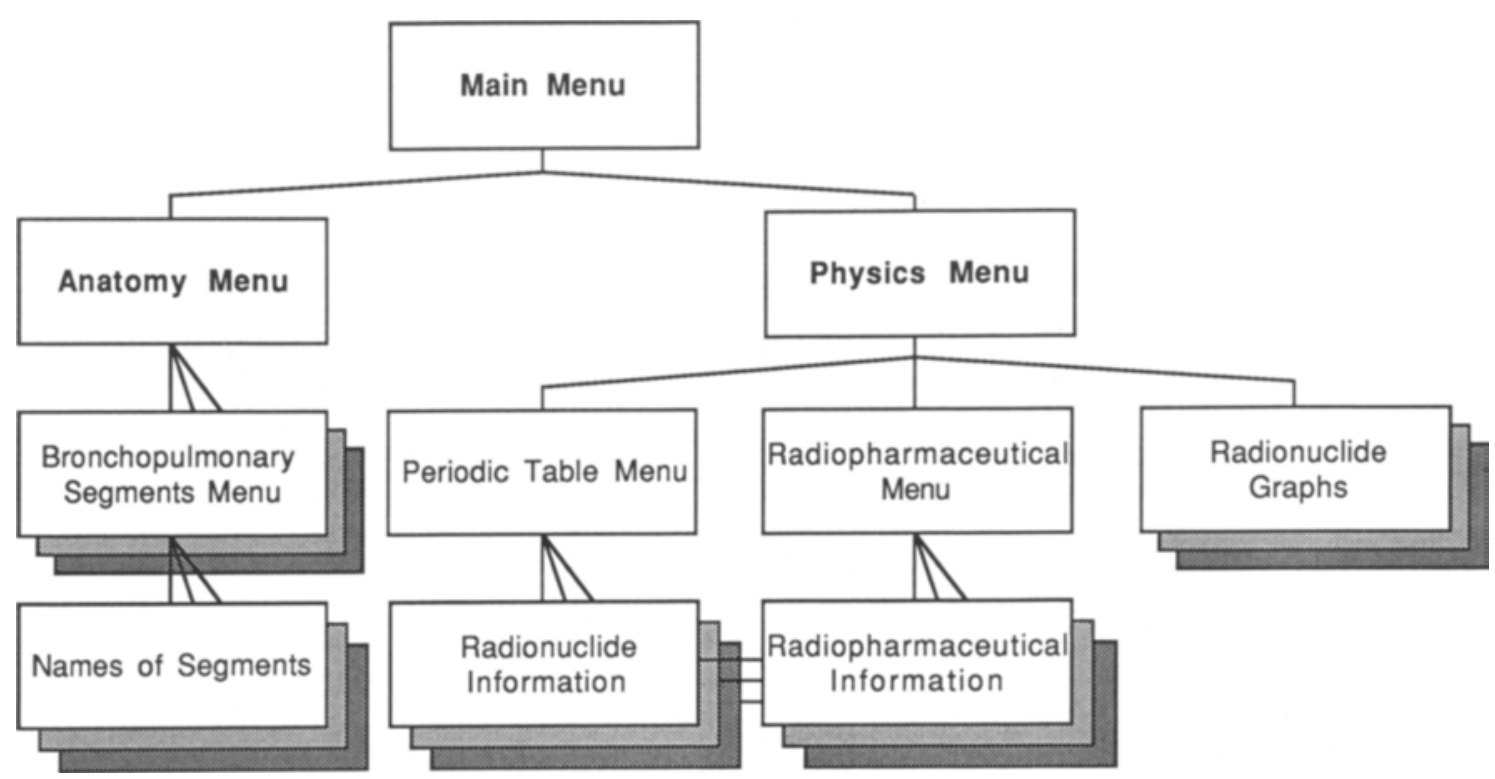

Fig 3. Overview of a portion of the hypertextbook. Each box represents a window of computer information. The lines between boxes represent links between each window. A group of three boxes represents a series of windows with information in the same format.

appears (Figure 5). Click the mouse on the lung of the human body drawing and the "Bronchopulmonary Segments" window appears (Figure 6). Click on one of the bronchopulmonary segments, and the name of the segment appears (Figure 7).

Figures 8 to 10 illustrate a search for information related to Tc-99m MAA (macroaggregated albumin). Figure 11 shows a graph of the major energies emitted by radionuclides.
This is just a brief overview of the hypertextbook related to pulmonary scintigraphy, and demonstrates how information can be presented in hypermedia format. Information related to other nuclear medicine procedures can be similarly provided, eg, a video sequence showing a time-activity curve being collected from a series of gated blood pool images. The hypertextbook at the moment is still a prototype, and development is continuing.

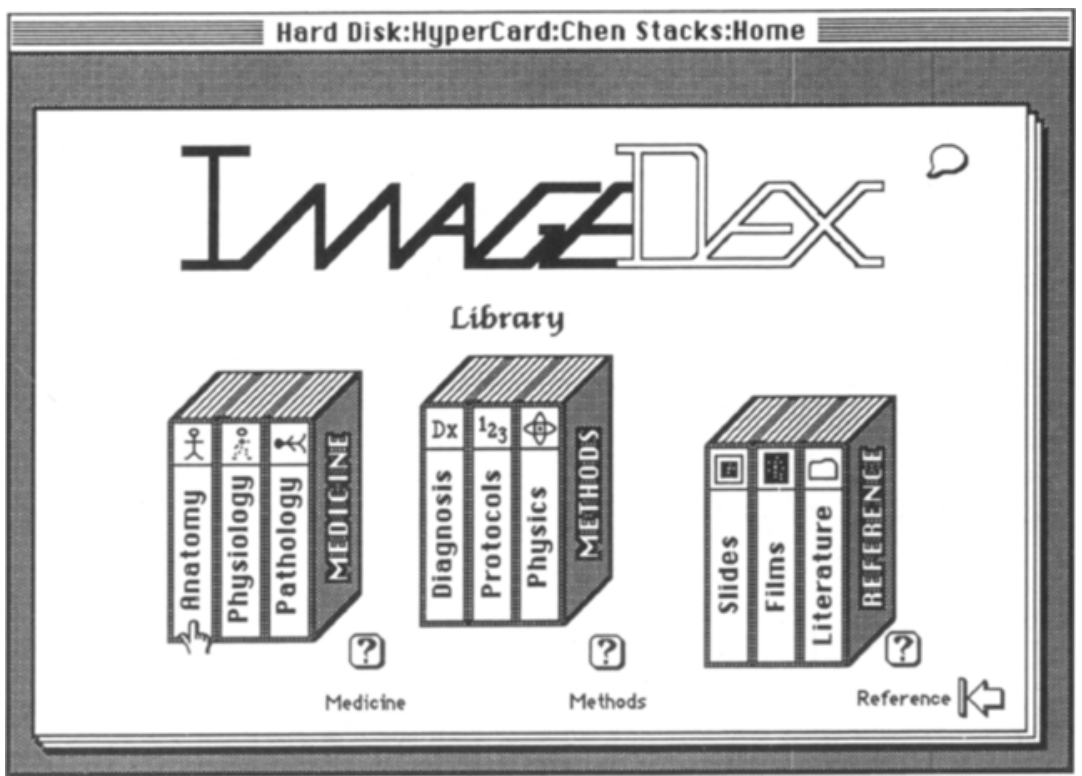

Fig 4. The "Main Menu" of the hypertextbook. Buttons on the menu can be activated with a computer mouse to branch off to more information. Here the "Anatomy" button is being activated. 
Fig 5. The "Anatomy" menu. Buttons on the drawing of the human body links to anatomic information of each organ system. Here the "Lung" button is being activated.

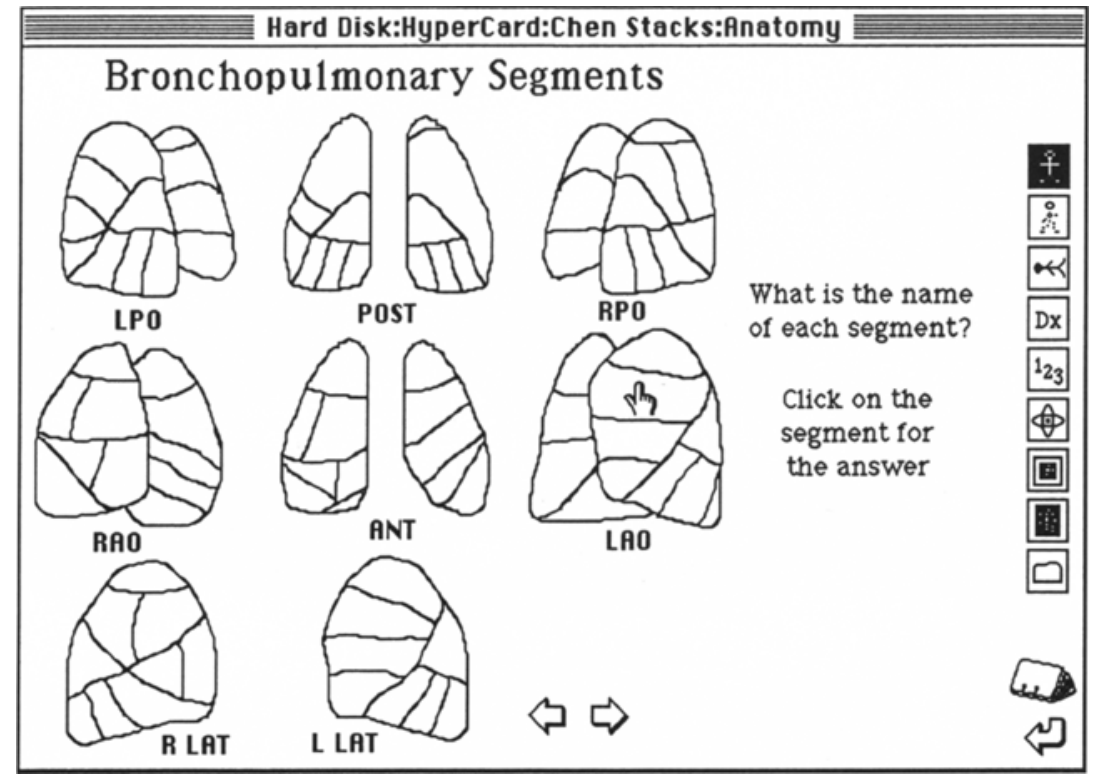

\section{ADVANTAGES AND DISADVANTAGES OF HYPERMEDIA}

An important feature of a hypermedia is that information is accessed in an interactive mode. This provides immediate feedback and personalized tutorials that are more meaningful and can provide long-term learning gains. ${ }^{4}$ Computerassisted instruction in radiology has been shown to be as effective as conventional lecture formats, and is subjectively rated superior by students because of its interactive nature. ${ }^{5}$

Microcomputers have been described as vehi- cles for continuing medical education. They make it possible for physicians to use educational programs at the home or office, without the need for expensive travel to distant courses and without loss of practice time. ${ }^{6}$ Hypermedia programs can decrease the need for direct interaction with a sonographer or a nuclear cardiologist which is necessary to become familiar with basic motion abnormalities and other findings related to dynamic images. Instead, an individual can consult a hypermedia textbook, such as the one previously described. Information provided in

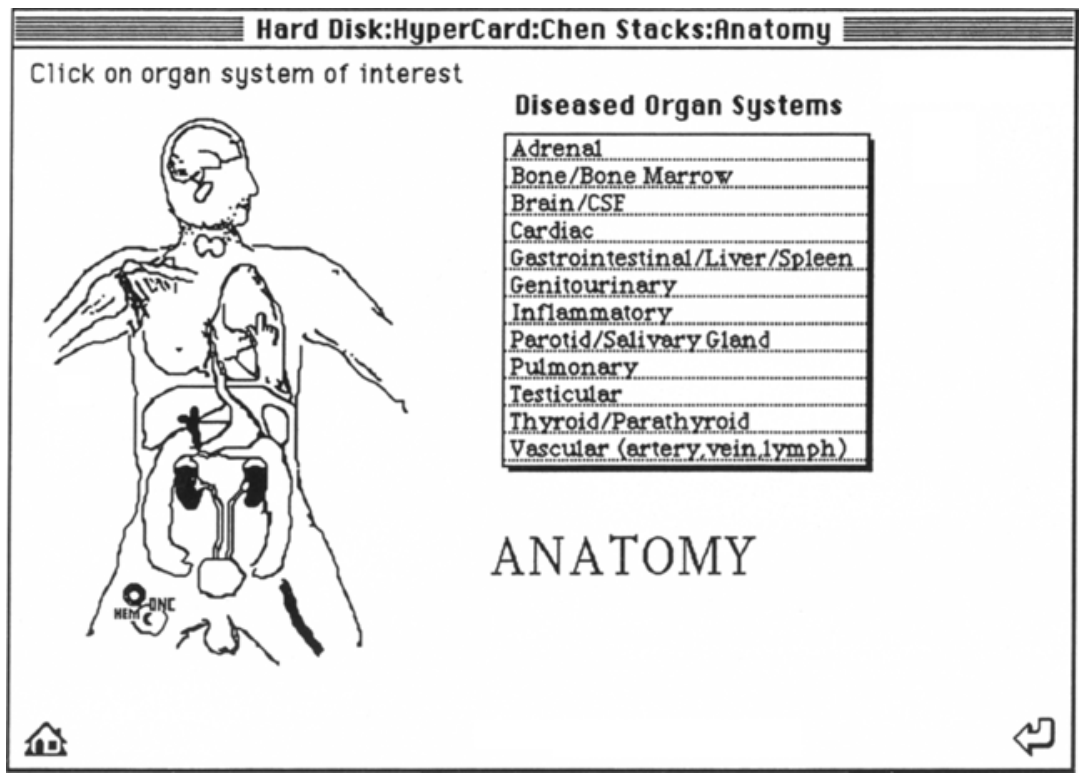

Fig 6. The "Bronchopulmonary Segments" window. A drawing showing the bronchopulmonary segments in multiple projections as seen on a lung scan. Here a button over a segment is being activated. 


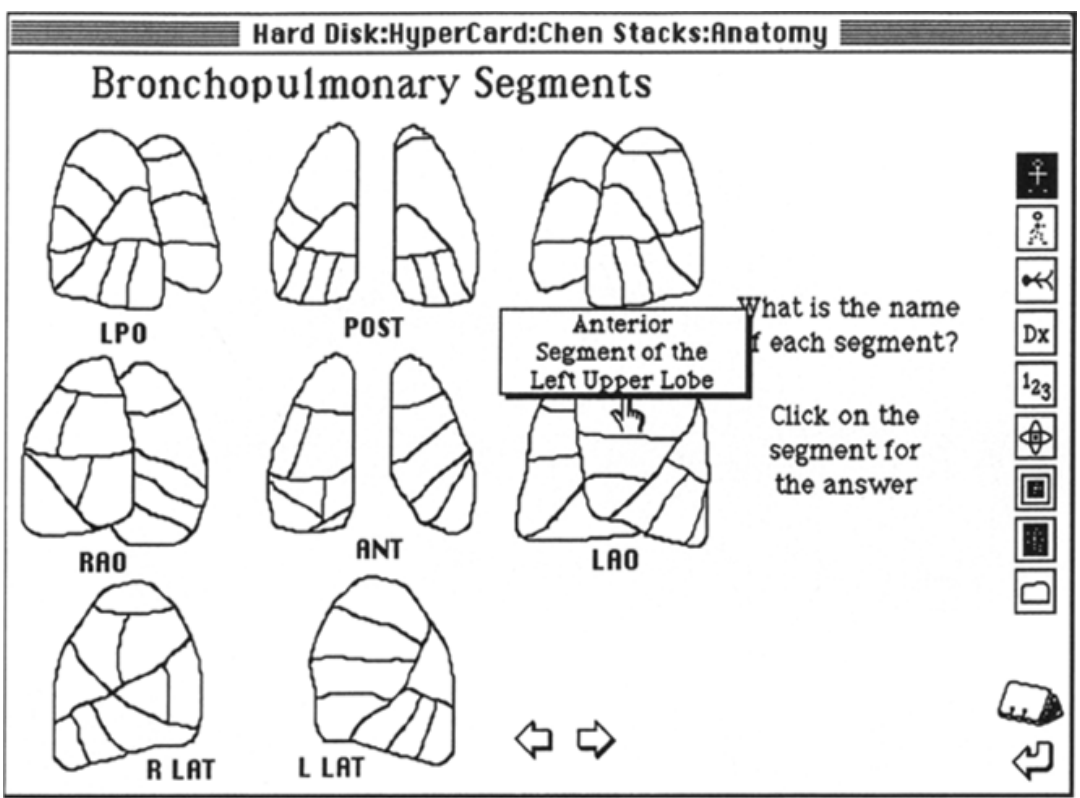

Fig 7. The "Bronchopulmonary Segments" window with the name of a segment visible, after the button overlying the segment has been activated.

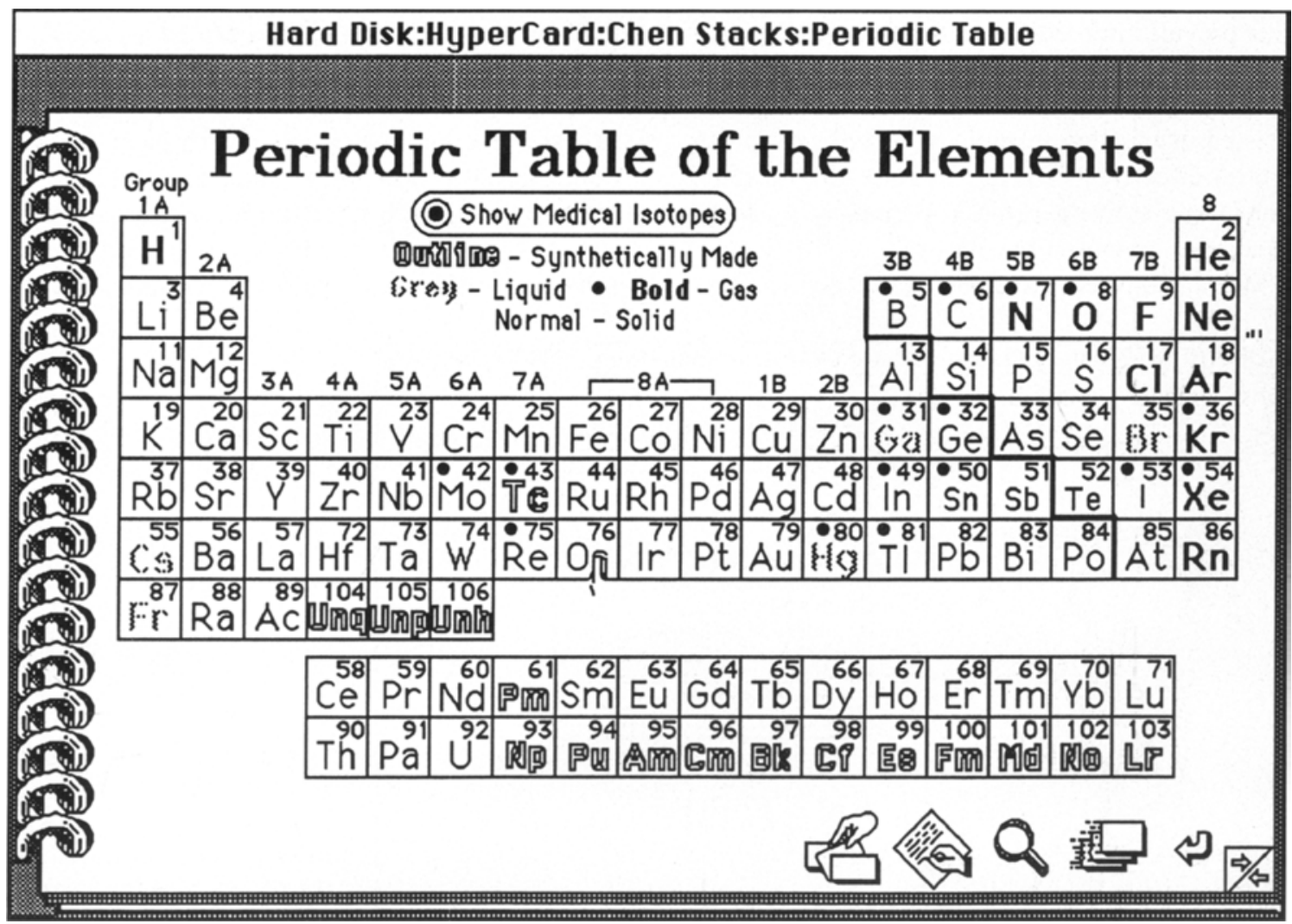

Fig 8. The "Periodic Table" Menu. Click on the element name technetium, "Tc," and the next window of information appears (see Fig 9). 


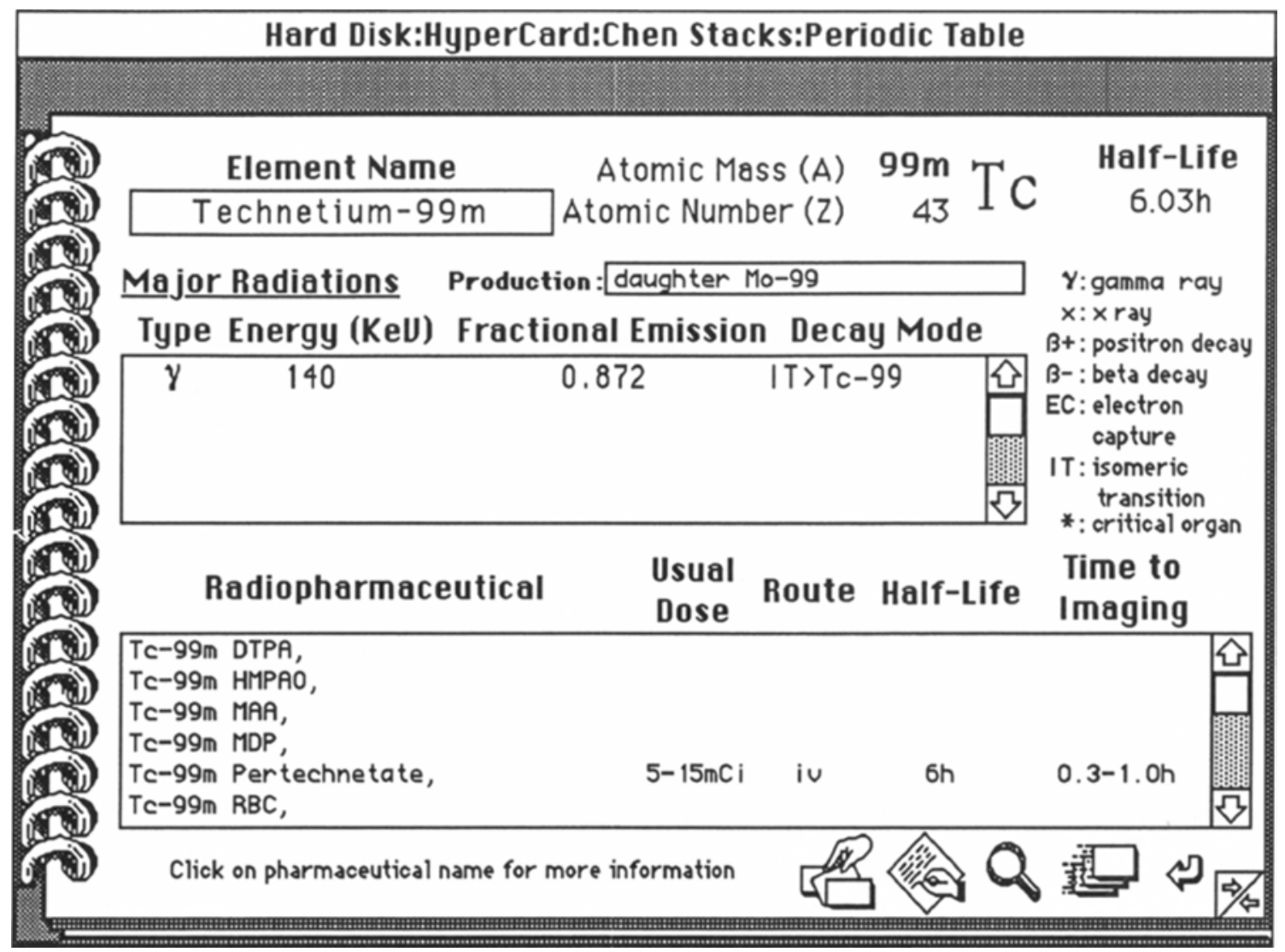

Fig 9. The "Tc-99m" window shows information pertinent to Tc-99m. Click on the words "Tc-99m MAA" and the next window appears (Figure 10).

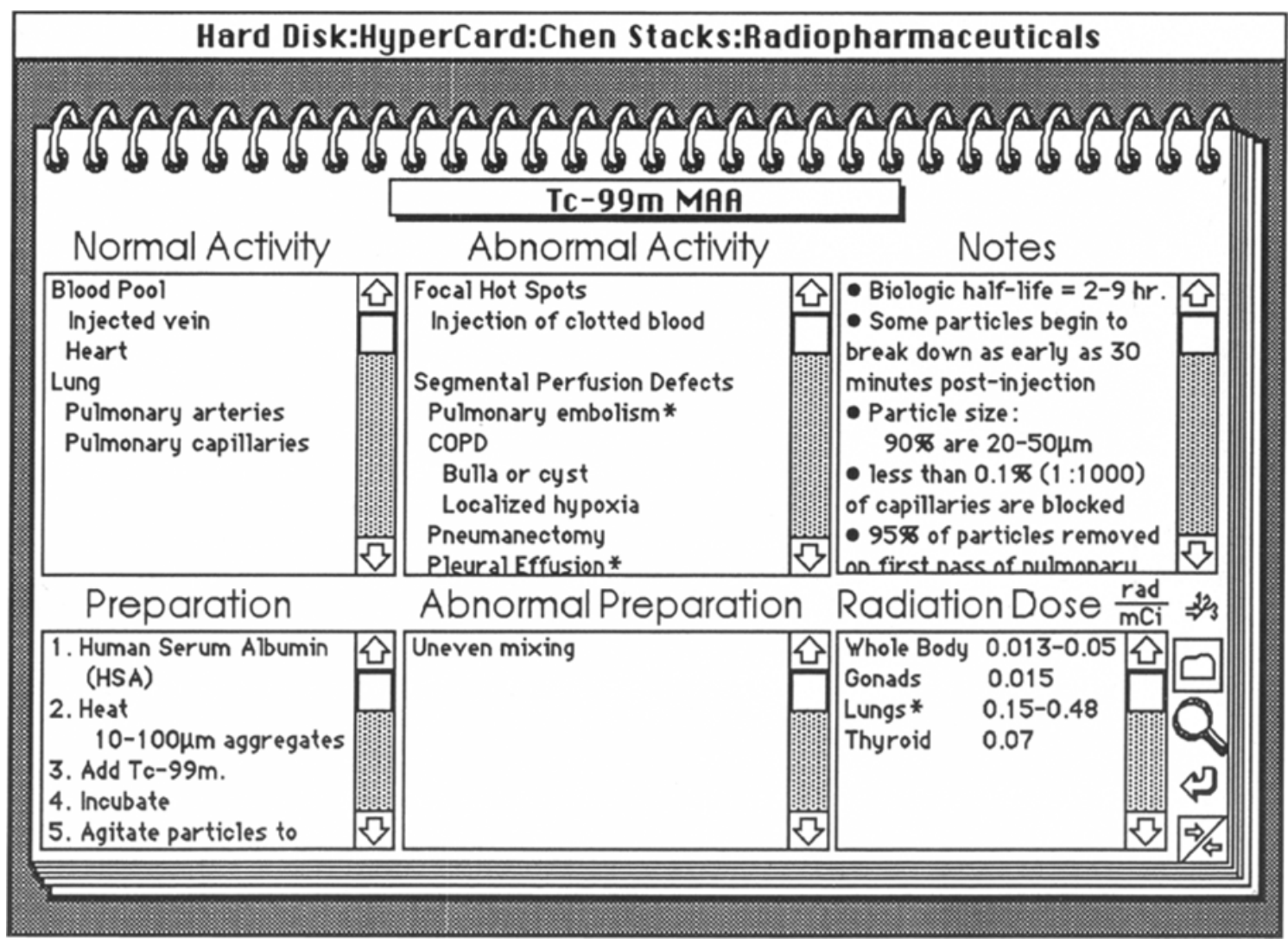

Fig 10. The "Tc-99m MAA" radiopharmaceutical information window. 


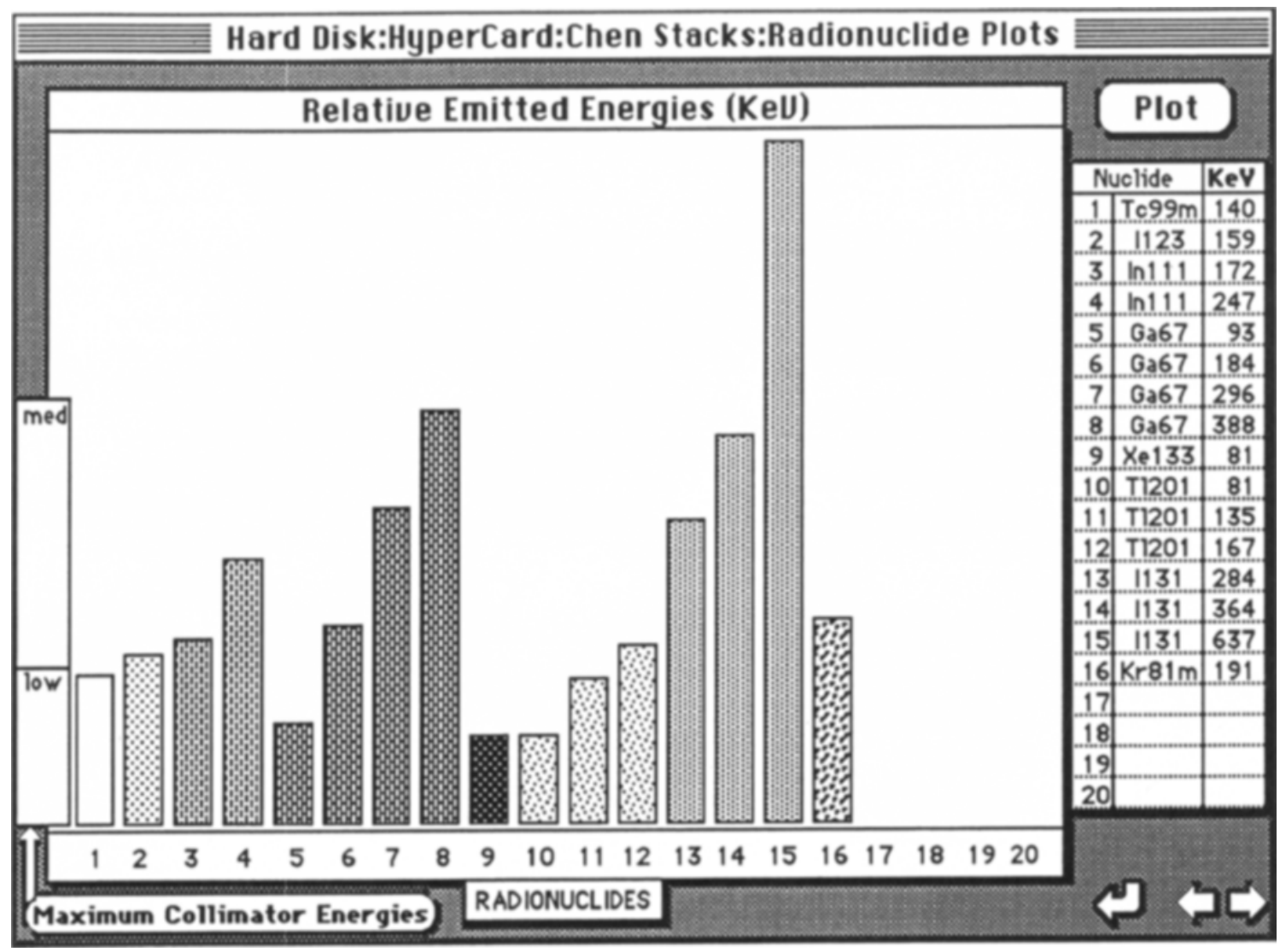

Fig 11. Radionuclide major emission energies graph. This is an interactive graph. Type in any radionuclide energy values in the table to the right, press the "Plot" button above the table, and the information is immediately plotted on the graph to the loft.

computer-based formats is also easier to update because the latest information is just inserted while replacing outdated information.

Hypermedia can provide more efficient computer-assisted access to a teaching collection library. A common problem with such libraries is the lack of sufficient organization. Typically, cases are separated only into sections indexed by American College of Radiology codes or by organ systems. However, the folders are then randomly placed within each section. A hypermedia database can be used to organize the library into a structured format with every case referenced to specific teaching points, and may be directly linked to the images themselves, after they have been transferred to a digitized format.

A summary of other advantages as noted by Conklin: ${ }^{2}$ (1) text can be organized in different ways depending on differing viewpoints; (2) it is quite natural to suspend reading temporarily along one line of investigation while looking into some detail, example, or related topic; (3) all references are easily followed forward or backward; (4) users can expand their own networks or annotate someone else's document; (5) both hierarchical and nonhierarchical organizations can be imposed on unstructured information, even multiple hierarchies can be used to organize the same material; (6) table of contents-style views support easier restructuring of large or complex documents, ie, global and local views can be mixed effectively; (7) text segments can be threaded iogether in many ways, allowing the same document to serve multiple functions; (8) since the same text segments can be referenced from several places, ideas can be expressed with less overlap and duplication; (9) references are embedded in their text, and if the text is moved, even to another document, the link information still provides direct access to the reference; and (10) several authors can collaborate, with the document and comments about the document tightly interwoven.

Another advantage is that information may be 
presented in ways that incorporate memory aids such as (1) multiple sense encoding, ie, items learned in picture form are more easily learned than an equivalent list of printed words; (2) searching for information leads to a deeper processing of the material and to stronger recall; (3) the principle of overviewing and mapping out a subject provides context and meaning and leads to the use of the single most powerful element in memory-visual memory; (4) the physical environment in which people learn can also have a profound influence on their ability to recall. Hypermedia presentations try to simulate familiar objects and suroundings, and (5) every new fact or concept learned adds to, and links up, with the existing network. When something new is encoded, it forms a link to the existing network and provides another hook onto which still more associations can be hanged or connected. The more that is learned and remembered, the greater the capacity for future learning and remembering. ${ }^{\text {? }}$
Disadvantages outlined by Conklin include: ${ }^{2}$ (1) disorientation or the tendency to lose location and direction in a nonlinear document; and (2) cognitive overhead, ie, the additional effort and concentration necessary to maintain several tasks or trails at one time. Another disadvantage is the need for a fast computer system supported by sufficiently powerful hypermedia software. Such systems may be costly, but are becoming more affordable with advances in computer technology.

\section{CONCLUSION}

A hypertextbook is one application of hypermedia in radiology. The hypertextbook previously described is still a prototype, but work to complete it is continuing. There are many advantages and some limitations to this method of computer-assisted education. However, with further development and use of hypermedia, this new medium can revolutionize the way radiologic knowledge is exchanged in the future.

\section{REFERENCES}

1. McLuhan M: Understanding Media. New York, McGraw-Hill, 1964

2. Conklin J: Hypertext: An introduction and survey. Computer 2:17-41, 1987

3. Goodman D: The Complete HyperCard Handbook. New York, Bantam, 1987

4. Aronberg DJ, Rodewald SS, Jost LG: Computerassisted instruction in radiology. Radiology 154:345-347, 1985
5. Jacoby CG, Smith WL, Albanese MA: An evaluation of computer-assisted instruction in radiology. AJR 143:675677,1984

6. Rosenblatt RA, Gaponoff M: The microcomputer as a vehicle for continuing medical education. J Fam Pract 18:629-632, 1984

7. Rose C: Accelerated Learning. New York, Dell, 1985 